\title{
两个新的化合物线四肽和单羟基甾醇的分离与结构鉴定
}

\author{
王长军梁远维廖小建＼cjkstart徐石海* \\ (暨南大学化学系 广州 510632)
}

\begin{abstract}
摘要 采用现代分离手段, 从采自中国南海碯洲岛的白穗软珊瑚 Nephthea sp.中分离纯化得到两个新的化合物分别为 线四肽化合物和单羟基甾醇, 经过 MS, ${ }^{1} \mathrm{H}$ NMR, ${ }^{13} \mathrm{C}$ NMR (DEPT), HSQC, HMBC 和 NOE 等光谱技术鉴定它们的结构 为: 亮氨酰- $N$-甲基亮氨酰-亮氨酰- $N$-甲基亮氨酸(1)和(20S,22R)-孕甾-1,4-二烯-22-乙酰基-3-酮(2), 这两个化合物均为 新化合物. 肿瘤活性实验表明两个新的化合物显示微弱的细胞毒性.

关键词 软珊瑚; Nephthea sp.; 线四肽; 单羟基甾醇
\end{abstract}

\section{Isolation and Identification of Two New Compounds Linear Tetrapeptide and Monohydroxysteroid}

\author{
Wang, Changjun Liang, Yuanwei Liao, Xiaojian Xu, Shihai* \\ (Department of Chemistry, Jinan University, Guangzhou 510632)
}

\begin{abstract}
Two new compounds have been isolated from Nephthea sp. collected from Naozhou Islands of the South China Sea. Their structures were established as leucyl- $N$-methyl-leucyl-leucyl- $N$-methyl-leucine (1), (20S,22R)-progesterone-1,4diene-22-acetyl-3-one (2) by MS, IR, ${ }^{1} \mathrm{H}$ NMR, ${ }^{13} \mathrm{C}$ NMR (DEPT), HSQC, HMBC and NOE analysis. Both of them are previously unknown compounds and showed weak cytotoxicity against the cancer cell lines.

Keywords coral; Nephthea sp.; linear tetrapeptide; monohydroxysteroid
\end{abstract}

海洋独特的生态系统是开发海洋药物的巨大宝库, 国际上现代海洋药物研究始于 20 世纪 60 年代, 取得了 一系列成就，已从海绵、海鞘、软珊瑚、软体动物、苔 藓虫、棘皮动物、海藻、细菌、真、微藻等各类海洋动 物中分离获得约 1500 种海洋产物, 具有生物碱、肽类、 大环内酯等各种结构类型，拥有抗肿瘤、抗病毒、抗炎 等各种生物活性 ${ }^{[1 \sim 7]}$. 葟肽是氨基酸连接数量小于 10 的 一种具有独特结构的小分子, 许多葟肽在人体中发挥着 不可替代的调节机能的作用. 自然界存在着大量具有生 物学活性的寡肽物质，它们涉及分子识别、信号传导、 细胞分化及个体发育等诸多领域, 为药物的开发提供又 一个途径 ${ }^{[811]}$. 甾醇是生物膜的重要组成部分和一些激 素的前体, 几乎存在于所有生物体内. 海洋珊瑚中存在 丰富的甾醇类化合物, 由于甾醇侧链结构的多样化和氧 化程度的不同, 使其结构较为复杂, 且具有广谱的生物 活性, 如抗肿瘤、抗真菌、抗菌、抗病毒和抗污染等 ${ }^{[2]}$.
我们在研究中国南海㖇洲岛软珊瑚 Nephthea sp.的抗肿 瘤活性成分时首次发现两个新的化合物: 亮氨 酰- $N$-甲 基亮氨酰-亮氨酰- $N$-甲基亮氨酸(1) 和 $(20 S, 22 R)$ - 孕 甾-1,4-二烯-22-乙酰基-3-酮(2)(图 1). 体外抗肿瘤体外 抗肿瘤活性实验表明, 两种化合物对肿瘤细胞 A549 和 Hepg2 表现出较弱的抑制作用.

\section{1 结果与讨论}

化合物 1: 无色油状, $[\alpha]_{\mathrm{D}}^{25}-66$ ( c $\left.0.15, \mathrm{CHCl}_{3}\right)$; HRESI-MS $m / z: 521.3679[\mathrm{M}+\mathrm{Na}]^{+}$给出该化合物的分 子式 $\mathrm{C}_{26} \mathrm{H}_{50} \mathrm{~N}_{4} \mathrm{O}_{5}$, 该分子式含有 4 个不饱和度. 将该化 合物进行盐酸解反应，喷酒 $0.2 \%$ 苑三酮试剂，在薄层板 上显紫红色和黄色两个斑点, 从而初步确定该化合物为 肽类化合物. 同时 ${ }^{1} \mathrm{H} N \mathrm{NMR}$ 和 ${ }^{13} \mathrm{C} \mathrm{NMR}$ 也表明该分子中 含有肽类化合物骨架. 为此, 只要对分子结构中羟基从 氢谱上分析可以确定有活泼氢 $\delta 10.27,7.29(\mathrm{br})$. 在 $\delta 4$

\footnotetext{
*E-mail: txush@jnu.edu.cn

Received July 2, 2011; revised November 1, 2011; accepted December 9, 2011.

Project supported by the National High Technology Development Project (No. 2006AA09Z408), the National Natural Science Foundation of China (Nos. 20772048, 21172094) and the Science and Technology Plan Project of Foundation Guangdong Province (No. 21172094).

国家 863 基金(No. 2006AA09Z408)、国家自然科学基金(Nos. 20772048, 21172094)和广东省科技计划(No. 211A080504007)资助项目.
} 

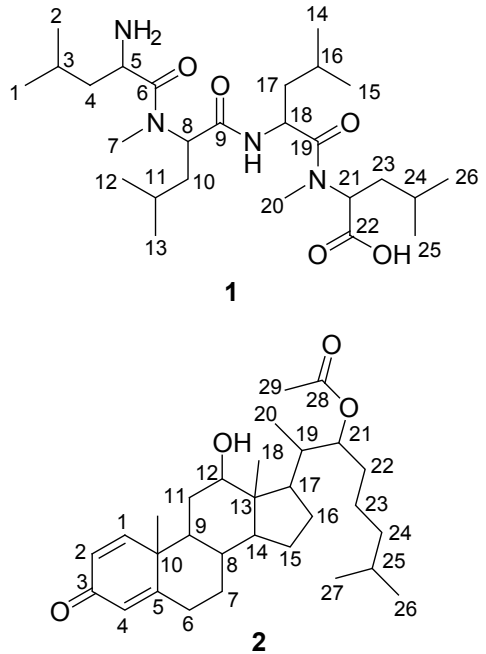

图 1 化合物 1 和 2 的结构

Figure 1 Structures of compounds $\mathbf{1}$ and $\mathbf{2}$

附近存在三组活泼氢. 全碳谱上分析可知有 23 个碳原 子. 在高场区存在大量的 $\mathrm{CH}_{3}, \mathrm{CH}_{2}(\delta 0.80 \sim 0.97,1.61 \sim$ 1.98)信号, 通过碳谱可知存在四个季碳 $(\delta 169.0,167.0$,
156.0，153.5)，三个亚甲基 $(\delta 44.98 ， 43.01 ， 42.55)$, 还有 三组活泼氢 $(\delta 4.15,3.95,3.78)$, 推测可能与氧原子相连. 在氢谱上分析有活泼氢 $(\delta 10.27)$ 可以确定推测应是羧酸 的氢原子. 四个羰基与一个羧基占用了 5 或 6 个氧原子, 通过上述分子式可以确定为 3 个羰基与一个羧基构成, 同时可以推断其余三组活泼氢应该与氮原子相连. 在氢 谱 $(\delta 7.30, \mathrm{br}$ s $)$ 和积分面积可以推断此处为 $\mathrm{NH}_{2}$, 从整体 判断该化合物为氨基酸一类化合物.

由 ${ }^{1} \mathrm{H}-{ }^{1} \mathrm{HCOSY}, \mathrm{HSQC}$ 和 $\mathrm{HMBC}$ 来归属分子片段的 连接情况: $\mathrm{H}-1$ 与 $\mathrm{H}-3, \mathrm{H}-2$ 与 $\mathrm{H}-3, \mathrm{H}-3$ 与 $\mathrm{H}-1, \mathrm{H}-2, \mathrm{H}-4$, $\mathrm{H}-5$ 与 $\mathrm{H}-4, \mathrm{H}-6$ 相关. 另一组 ${ }^{1} \mathrm{H}-{ }^{1} \mathrm{H}$ COSY 为 $\mathrm{H}-8$ 与 H-10, H-10 与 $\mathrm{H}-8, \mathrm{H}-11, \mathrm{H}-11$ 与 $\mathrm{H}-10, \mathrm{H}-12$ 和 $\mathrm{H}-13$ 相 关. HMBC 可知连接情况: C-3 与 H-1, H-2, H-4 相关, C-5 与 $\mathrm{H}-4, \mathrm{H}-3$ 相关, C-10 与, H-8, H-11 相关, C-11 与 H-10, H-12, H-13 相关，可推测分子片段 $\mathrm{CH}_{3}(1,2)-\mathrm{CH}(3)-$ $\mathrm{CH}_{2}$ (4)-CH(5)存在, $\mathrm{H}-5$ ( $\delta 3.78, \mathrm{dd}, J=8.3,4.8 \mathrm{~Hz}$ )由上 部分推论可知 C-5 与氮原子相连. H-7 $(\delta 2.92, \mathrm{~s}), \mathrm{C}-7$ ( $\delta$ $\left.33.46, \mathrm{CH}_{3}\right), \mathrm{H}-8(\delta 3.97, \mathrm{t}, J=10.6 \mathrm{~Hz}), \mathrm{C}-8(\delta 60.40$, $\mathrm{CH}), \mathrm{C}-7$ 和 C-8 推断与氮原子相连. C-6 ( $\delta 156.00)$, 羰基

表 1 化合物 1 的相关核磁数据 $\left(\mathrm{CDCl}_{3}\right.$ 为溶剂 $)$

Table 1 NMR data of 1 in $\mathrm{CDCl}_{3}$

\begin{tabular}{|c|c|c|c|c|}
\hline Position & $\delta_{\mathrm{C}}$ & $\delta_{\mathrm{H}}$ & COSY & HMBC \\
\hline 1 & $20.92\left(\mathrm{CH}_{3}\right)$ & $0.89 \sim 0.92(\mathrm{~m})$ & $\mathrm{H}-3$ & \\
\hline 2 & $21.93\left(\mathrm{CH}_{3}\right)$ & $0.89 \sim 0.92(\mathrm{~m})$ & $\mathrm{H}-3$ & \\
\hline 3 & $23,34(\mathrm{CH})$ & $1.83 \sim 1.85(\mathrm{~m})$ & H-1, H-2 & $\mathrm{C}-1, \mathrm{C}-2, \mathrm{C}-4$ \\
\hline 4 & $44.98\left(\mathrm{CH}_{2}\right)$ & $1.51 \sim 1.54(\mathrm{~m})$ & H-3, H-5 & $\mathrm{C}-3, \mathrm{C}-5$ \\
\hline 5 & $53.97(\mathrm{CH})$ & $3.78(\mathrm{dd}, J=8.3,4.8 \mathrm{~Hz})$ & $\mathrm{H}-4$ & $\mathrm{C}-3, \mathrm{C}-4$ \\
\hline 6 & $156.00(\mathrm{C})$ & & & \\
\hline 7 & $33.46\left(\mathrm{CH}_{3}\right)$ & $2.92(\mathrm{~s})$ & & \\
\hline 8 & $60.40,(\mathrm{CH})$ & $3.97(\mathrm{t}, J=10.6 \mathrm{~Hz})$ & $\mathrm{H}-10$ & C-9, C-10 \\
\hline 9 & $169.00(\mathrm{C})$ & & & C-8, C-10, \\
\hline 10 & $43.10\left(\mathrm{CH}_{2}\right)$ & $1.75 \sim 1.77(\mathrm{~m})$ & $\mathrm{H}-11$ & C-8, C-9, C-11 \\
\hline 11 & $24.85(\mathrm{CH})$ & $1.68 \sim 1.69(\mathrm{~m})$ & $\mathrm{H}-12, \mathrm{H}-13$ & $\mathrm{C}-10, \mathrm{C}-12, \mathrm{C}-13$ \\
\hline 12 & $23.22\left(\mathrm{CH}_{3}\right)$ & $0.89 \sim 0.92(\mathrm{~m})$ & & \\
\hline 13 & $24.21\left(\mathrm{CH}_{3}\right)$ & $0.89 \sim 0.92(\mathrm{~m})$ & & $\begin{array}{l}\text { C-8, C-10, } \\
\text { C-11, C-12 }\end{array}$ \\
\hline 14 & $22.02\left(\mathrm{CH}_{3}\right)$ & $0.89 \sim 0.92(\mathrm{~m})$ & H-16 & \\
\hline 15 & $23.18\left(\mathrm{CH}_{3}\right)$ & $0.89 \sim 0.92(\mathrm{~m})$ & H-16 & C-15, C-16, C-17 \\
\hline 16 & $24.21(\mathrm{CH})$ & $1.59 \sim 1.61(\mathrm{~m})$ & H-14, H-15 & C-14, C-15, C-17 \\
\hline 17 & $42.55\left(\mathrm{CH}_{2}\right)$ & $1.75 \sim 1.76(\mathrm{~m})$ & H-16, H-18 & \\
\hline 18 & $60.40(\mathrm{CH})$ & $3.94(\mathrm{t}, J=10.6 \mathrm{~Hz})$ & $\mathrm{H}-17$ & \\
\hline 19 & $153.50(\mathrm{C})$ & & & \\
\hline 20 & $33.10\left(\mathrm{CH}_{3}\right)$ & $2.92(\mathrm{~s})$ & & \\
\hline 21 & $62.50(\mathrm{CH})$ & $4.15(\mathrm{t}, J=6.0 \mathrm{~Hz})$ & $\mathrm{H}-23$ & \\
\hline 22 & $167.00(\mathrm{C})$ & & & \\
\hline 23 & $43.10\left(\mathrm{CH}_{2}\right)$ & $1.74 \sim 1.76(\mathrm{~m})$ & H-21, H-24 & $\begin{array}{l}\text { C-21, C-24, } \\
\text { C-25, C-26 }\end{array}$ \\
\hline 24 & $24.85(\mathrm{CH})$ & $1.71 \sim 1.74(\mathrm{~m})$ & H-25, H-26 & C-23, C-25, C-26 \\
\hline 25 & $24.34\left(\mathrm{CH}_{3}\right)$ & $0.89 \sim 0.92(\mathrm{~m})$ & H-24 & \\
\hline 26 & $24.85\left(\mathrm{CH}_{3}\right)$ & $0.89 \sim 0.92(\mathrm{~m})$ & H-26 & \\
\hline
\end{tabular}


碳的化学位移在 $\delta 190 \sim 210$, 酰胺碳化学位移在 $\delta$ $150 \sim 170$, 可以确定 C-6 为酰胺键, 再通过 ${ }^{1} \mathrm{H}-{ }^{1} \mathrm{H}$ COSY 和 $\mathrm{HMBC}$ 可以推出化合物 $\mathbf{1}$ 片段, 对照分析化合物 $\mathbf{1}$ 分子式: $\mathrm{C}_{26} \mathrm{H}_{50} \mathrm{~N}_{4} \mathrm{O}_{5}$ 和二维核磁数据分析可知, 该化合 物另一片段连接关系与图 2 相同. 最终确定该化合物平 面结构为线亮氨酰- $N$-甲基亮氨酰-亮氨酰- $N$-甲基亮氨 酸.

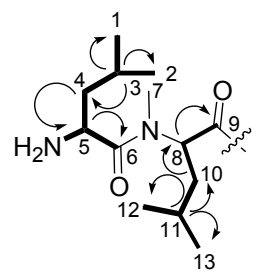

图 2 化合物 1 片段的 ${ }^{1} \mathrm{H}-{ }^{1} \mathrm{H}$ COSY 与 $\mathrm{HMBC}$ 的主要相关关 系

Figure $2{ }^{1} \mathrm{H}-{ }^{1} \mathrm{H}$ COSY and selected key HMBC correlations of compound 1 fragment

化合物 2: 淡黄色色油状, $[\alpha]_{\mathrm{D}}^{25}+46$ (c 0.11 , $\mathrm{CHCl}_{3}$ ); HRESI-MS m/z $493.3086[\mathrm{M}+\mathrm{Na}]^{+}$给出该化合 物的分子式 $\mathrm{C}_{30} \mathrm{H}_{46} \mathrm{O}_{4}$, 该分子式含有 8 个不饱和度. ${ }^{13} \mathrm{C}$ NMR 和 DEPT 谱显示 29 个碳信号, 分别为 6 个 $\mathrm{CH}_{3}, 8$ 个 $\mathrm{CH}_{2}, 11$ 个 $\mathrm{CH}$ 和 5 个 $\mathrm{C}$; 通过 DQF-COSY, HSQC,
$\mathrm{HMBC}$ 逐个对 C, H 信号进行归属(表 2). ${ }^{13} \mathrm{C} \mathrm{NMR}$ 和 ${ }^{1} \mathrm{H}$ NMR 低场区 $\delta 186.3(\mathrm{C}), 155.2(\mathrm{CH}), 127.7(\mathrm{CH})$ 及 124.1 $(\mathrm{CH})$ 与 3 个烯氢信号 $[\delta 7.02(\mathrm{~d}, J=10.1 \mathrm{~Hz}, 1 \mathrm{H}, \mathrm{H}-1)$, 6.22 (dd, $J=10.1,1.7 \mathrm{~Hz}, 1 \mathrm{H}, \mathrm{H}-2), 6.06$ (s, 1H, H-4)]信 号说明该甾醇存在羰基与两个双键共轭.

${ }^{1} \mathrm{H}$ NMR 谱中显示化合物 2 有 6 个甲基氢信号 $[\delta$ 0.75 (s, 3H, Me-18), 1.20 (s, 3H, Me-19), 1.06 (s, 3H, Me-21), 0.85 (s, 3H, Me-27), 0.85 (s, 3H, Me-28), 0.87 (s, $3 \mathrm{H}, \mathrm{Me}-30)], 3$ 个烯氢信号 $[\delta 7.02(\mathrm{~d}, J=10.1 \mathrm{~Hz}, 1 \mathrm{H}$, H-1), 6.22 (dd, $J=10.1,1.7 \mathrm{~Hz}, 1 \mathrm{H}, \mathrm{H}-2), 6.06$ (s, 1H, $\mathrm{H}-4)]$ 和 2 个连氧碳上氢的信号 $[\delta 4.89(\mathrm{~m}), 3.74(\mathrm{dd}, J=$ $11.0,3.7 \mathrm{~Hz}), 4.68(\mathrm{~m})]$, 再通过碳谱数据表明该分子是 符合孕甾醇类化合物特征 ${ }^{[13]}$ ，同时对 Lieberman-Burchard 试剂呈阳性反应，也可表明它是甾醇类化合物.

${ }^{13} \mathrm{C} N M R$ 谱中, 可以推出化合物 2 含有 30 个碳原 子, 结合 DEPT 谱显示含有 6 个甲基 $(\delta 7.45,14.57,18.53$, $21.37,22.43 ， 22.71) ， 8$ 个亚甲基 $(\delta 23.69 ， 26.14 ， 26.83$ ， $27.93,29.66,32.71,32.90,32.96), 11$ 个次甲基信号 $(\delta$ $27.93,34.43,37.37,50.52$, 53.16, 54.21, 76.70, 77.70, $124.1 ， 127.7,155.2)$ 和 5 个季碳原子 $(\delta 43.16,48.33$, 168.6, 171.1，186.3). 再根据 $\mathrm{HMQC}$ 谱逐个对 ${ }^{13} \mathrm{C} \mathrm{NMR}$ 和 ${ }^{1} \mathrm{H}$ NMR 进行了归属(表 2).

表 2 化合物 2 的相关核磁数据 $\left(\mathrm{CDCl}_{3}\right.$ 为溶剂 $)$

Table 2 NMR data of 2 in $\mathrm{CDCl}_{3}$

\begin{tabular}{|c|c|c|c|c|}
\hline Position & $\delta_{\mathrm{C}}$ & $\delta_{\mathrm{H}}$ & COSY & HMBC \\
\hline 1 & $155.2(\mathrm{CH})$ & $7.02(\mathrm{~d}, J=10.1 \mathrm{~Hz})$ & $\mathrm{H}-2$ & $\mathrm{C}-2, \mathrm{C}-3$ \\
\hline 2 & $127.7(\mathrm{CH})$ & $6.22(\mathrm{dd}, J=10.1,1.7 \mathrm{~Hz})$ & $\mathrm{H}-1$ & \\
\hline 3 & $186.3(\mathrm{C})$ & & & \\
\hline 4 & $124.1(\mathrm{CH})$ & $6.06(\mathrm{~s})$ & & $\mathrm{C}-2, \mathrm{C}-6$ \\
\hline 5 & $156.00(\mathrm{C})$ & & & \\
\hline 6 & $32.96\left(\mathrm{CH}_{2}\right)$ & $1.65 \sim 1.67(\mathrm{~m})$ & $\mathrm{H}-7$ & \\
\hline 7 & $29.66\left(\mathrm{CH}_{2}\right)$ & $1.49 \sim 1.51(\mathrm{~m})$ & $\mathrm{H}-14$ & $\mathrm{C}-7, \mathrm{C}-9, \mathrm{C}-14$ \\
\hline 8 & $34.43(\mathrm{CH})$ & $1.53 \sim 1.55(\mathrm{~m})$ & $\mathrm{H}-10$ & \\
\hline 9 & $53.16(\mathrm{C})$ & $0.91 \sim 0.94(\mathrm{~m})$ & & \\
\hline 10 & $48.30(\mathrm{C})$ & & & \\
\hline 11 & $32.90(\mathrm{CH})$ & $1.65 \sim 1.67(\mathrm{~m})$ & $\mathrm{H}-9, \mathrm{H}-12$ & \\
\hline 12 & $76.70(\mathrm{CH})$ & $3.38(\mathrm{dd}, J=10.8,4.6 \mathrm{~Hz})$ & $\mathrm{H}-11$ & \\
\hline 13 & $43.20(\mathrm{C})$ & & & \\
\hline 14 & $54.20(\mathrm{CH})$ & $1.31 \sim 1.33(\mathrm{~m})$ & $\mathrm{H}-8$ & $\mathrm{C}-8, \mathrm{C}-13, \mathrm{C}-15$ \\
\hline 15 & $23.69\left(\mathrm{CH}_{2}\right)$ & $1.60 \sim 1.61(\mathrm{~m})$ & & \\
\hline 16 & $26.83\left(\mathrm{CH}_{2}\right)$ & $1.75 \sim 1.78(\mathrm{~m})$ & & \\
\hline 17 & $50.50(\mathrm{CH})$ & $1.04 \sim 1.06(\mathrm{~m})$ & & \\
\hline 18 & $7.50\left(\mathrm{CH}_{3}\right)$ & $0.75(\mathrm{~s})$ & & $\mathrm{C}-12, \mathrm{C}-13, \mathrm{C}-14, \mathrm{C}-17$ \\
\hline 19 & $18.50\left(\mathrm{CH}_{3}\right)$ & $1.16 \sim 1.20,(\mathrm{~m})$ & & C-1, C-5, C-9, C-10 \\
\hline 20 & $37.40(\mathrm{CH})$ & $1.78 \sim 1.81(\mathrm{~m})$ & & C-17, C-21, C-22 \\
\hline 21 & $14.60\left(\mathrm{CH}_{3}\right)$ & $1.04 \sim 1.06(\mathrm{~m})$ & & C-17, C-20, C-22 \\
\hline 22 & $77.01(\mathrm{CH})$ & $4.87 \sim 4.89(\mathrm{~m})$ & $\mathrm{H}-23$ & $\mathrm{C}-20, \mathrm{C}-23, \mathrm{C}-30$ \\
\hline 23 & $32.71\left(\mathrm{CH}_{2}\right)$ & $1.40 \sim 1.42(\mathrm{~m})$ & H-22 & \\
\hline 24 & $26.14(\mathrm{CH})$ & $2.57(\mathrm{td}, J=13.5,4.8 \mathrm{~Hz})$ & & \\
\hline
\end{tabular}




\begin{tabular}{ccccc}
\hline \multicolumn{1}{c}{} & & 续表 \\
\hline Position & $\delta_{\mathrm{C}}$ & $\delta_{\mathrm{H}}$ & $\mathrm{COSY}$ & HMBC \\
\hline 25 & $35.48\left(\mathrm{CH}_{2}\right)$ & $1.25 \sim 1.27(\mathrm{~m})$ & $\mathrm{H}-24$ & $\mathrm{C}-25, \mathrm{C}-27, \mathrm{C}-28$ \\
26 & $27.90(\mathrm{CH})$ & $1.45 \sim 1.47(\mathrm{~m})$ & $\mathrm{H}-26$ & $\mathrm{C}-26$ \\
27 & $22.43\left(\mathrm{CH}_{3}\right)$ & $0.85 \sim 0.87(\mathrm{~m})$ & $\mathrm{H}-26$ & $\mathrm{C}-27$ \\
28 & $22.71\left(\mathrm{CH}_{3}\right)$ & $0.85 \sim 0.87(\mathrm{~m})$ & $\mathrm{H}-26$ & \\
29 & $171.1(\mathrm{C})$ & & & \\
30 & $21.40\left(\mathrm{CH}_{3}\right)$ & $2.03(\mathrm{~s})$ & & \\
\hline
\end{tabular}

HMBC 谱上显示如下的相关关系: H-19 和 C-1, C-5, C-9, C-10 有相关, H-1 和 C-2, C-3, C-5 有相关, H-3 和 $\mathrm{C}-6$ 相关, ${ }^{1} \mathrm{H}-{ }^{1} \mathrm{H}$ COSY 中, $\mathrm{H}-1$ 与 $\mathrm{H}-2$ 有相关, $\mathrm{H}-6$ 与 $\mathrm{H}-7$ 有相关, H-7 和 H-8 有相关, H-8 和 H-9 有相关. 由这些 相关关系, 可以确定 $\mathrm{A}$ 和 $\mathrm{B}$ 环片段关系, 并确定双键与 羰基共轭部分. HMBC 还显示有以下信号: $\mathrm{H}-18$ 和 C-12, C-13，C-14， C-17 有相关, H-17 和 C-13, C-18 有相关; $\mathrm{H}-12$ 与 $\mathrm{C}-17, \mathrm{C}-18$ 有相关, $\mathrm{H}-16$ 与 $\mathrm{C}-13$ 有相关; ${ }^{1} \mathrm{H}-{ }^{1} \mathrm{H}$ COSY 中, H-11 与 $\mathrm{H}-12$ 有相关, H-11 和 H-9 有相关, H-9 和 H-8 有相关, H-15 与 $\mathrm{H}-16$ 有相关, H-16 与 $\mathrm{H}-17$ 有相 关，通过这些关系得到 C-11，C-12，C-13，C-14，C-15, C-16, C-17 和 C-18 的归属信息. 因此推出甾醇 C, D 环 片段, 这样就可以确定 C-12 上羟基的位置. 对于化合物 的支链部分, $\mathrm{HMBC}$ 中 $\mathrm{H}-26$ 和 C- $25, \mathrm{C}-27$ 及 $\mathrm{C}-28$ 有相 关; H-22 和 C-20, C-23, C-30 有相关; 另外, H-24 和 C-22, C-25, C-26, C-27 有相关, H-23 与 C-20, C-22, C- 25 有相 关, H-20 与 $\mathrm{H}-21$ 有相关, $\mathrm{H}-20$ 与 $\mathrm{H}-22$ 有相关, $\mathrm{H}-23$ 与 $\mathrm{H}-24$ 相关, $\mathrm{H}-24$ 与 $\mathrm{H}-25$, 以上相关关系可以得到 C-20, C-21, C-23, C-24, C-25. C-26 的归属信息, 从而确定 22 位酯基位置, 支链的片段结构如下, 因此, 将图 3 中 2.1, 2.2 和 2.3 连起来, 就构成了化合物 $\mathbf{2}$ 的平面结构.
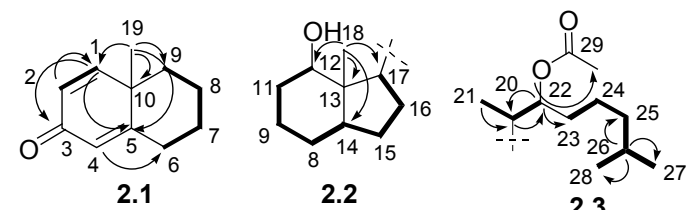

图 3 化合物 2 片段的 ${ }^{1} \mathrm{H}-{ }^{1} \mathrm{H}$ COSY 与 $\mathrm{HMBC}$ 的主要相关关 系

Figure $3 \quad{ }^{1} \mathrm{H}-{ }^{1} \mathrm{H}$ COSY and selected key HMBC correlations of compound 2 fargment

化合物 2 的空间结构可以通过 NOESY 谱来确定. 甾核的四个环处于反式构型, H-9 和 H-12, H-12 和 H-14 有相关说明 $\mathrm{OH}-12$ 是 $\beta$ 构型. 对于 C-20 位的构型, 在 NOE 中可以看到 H-2 2 与 $\mathrm{H}-16 \alpha$ 有相关, 可以得出 C-20 位为 $S$ 构型. 通过与文献化合物 $2-1^{[14]}$ 、文献化合物 $2-2^{[15]}$ 对照, C-17, C-20, C-21 化学位移数值基本相近, 故 鉴定化合物 2 中 C-20为 $S$ 构型. 综上所述, 确定化合物
2 的结构为 (20S,22R)-孕甾-1,4-二烯-22-乙酰基-3-酮.

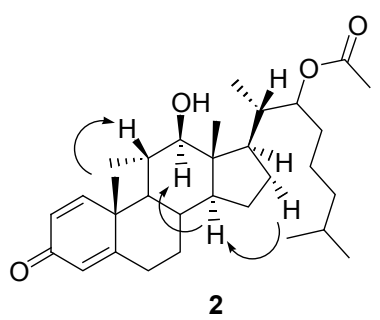

图 4 化合物 2 的 NOE 的主要相关关系

Figure 4 Selected key NOESY correlations of compound 2

\section{2 实验部分}

\section{1 仪器与试剂}

NICOLET 5DX-FT 红外光谱仪; Bruker AvanceIII400 型核磁共振仪; 高分辨质谱仪 MAT95XP; 液相色 谱一质谱联用仪 LCQ DECA XP; Aglient-1200 液相色谱; 全自动酶标仪 BIORAD-680; XDS-1B 显微镜; 不锈钢手 提式压力蒸汽灭菌器; 国产显微熔点仪, 温度计未校正; 薄层层析及柱层析用硅胶 $\mathrm{H}(40 \mu \mathrm{m})$, 青岛海洋化工厂 生产. 原材料采集于中国南海砶洲岛, 编号为 0701-01, 种属由荷兰阿姆斯特丹大学 Rob van Soest 教授鉴定.

\section{2 提取分离}

将软珊瑚 Nephthea sp. (湿重 $1.0 \mathrm{~kg}$ ) 用水泡洗后剪 碎, 用 $95 \%$ 乙醇在室温下浸泡 $10 \mathrm{~d} \times 5$, 合并浸泡液, 减 压浓缩得褐色膏状物后, 用乙酸乙酯溶解亳状物. 溶解 部分用乙酸乙酯-水分配 10 次, 合并乙酸乙酯相, 并减 压浓缩得褐色膏状物 $20.3 \mathrm{~g}$. 体外抗肿瘤活性测试表明 该膏状物对肿瘤细胞 BEL-7402, Hela 和 MCF 具有一定 的抑制作用, $\mathrm{IC}_{50}$ 值分别为 $3.05,1.59$ 和 $16.81 \mu \mathrm{g} / \mathrm{mL}$. 膏 状物用 300 400 目 Flash 硅胶进行快速柱层析，用极性 不断增加的石油醚一乙酸乙酯体系混合液洗脱，50\%石 油醚-乙酸乙酯洗脱出亮橙红色组分 $\mathrm{A}$, 固体 $\mathrm{A}$ 多次硅 胶快速柱层析, 凝胶柱反复柱层析, 最后通过 Agilent 1200 多次纯化, HPLC 条件: $V$ (乙腈) $/ V$ (水) $=85 / 15$, 时间 为 $50 \mathrm{~min}$, Phenomenex C18 $250 \mathrm{~mm} \times 4.6 \mathrm{~mm}$ 色谱柱得 到化合物亮氨酸- $N$-甲基亮氨酸-亮氨酸- $N$-甲基亮氨酸 (1) $6.8 \mathrm{mg}$ 和(20S,22R)-孕甾-1,4-二烯-22-乙酰基-3 酮(2) 
$5.2 \mathrm{mg}$.

化合物 1: 无色油状, $[\alpha]_{\mathrm{D}}^{25}-66\left(c 0.15, \mathrm{CHCl}_{3}\right)$; IR (KBr) $v_{\max }: 3351,3305,2957,1638$; HRESI-MS $\mathrm{m} / \mathrm{z}$ : $521.3679[\mathrm{M}+\mathrm{Na}]^{+}$(calcd for $\mathrm{C}_{26} \mathrm{H}_{50} \mathrm{~N}_{4} \mathrm{O}_{5} 498.2680$ ). ${ }^{1} \mathrm{H}$ $\operatorname{NMR}\left(\mathrm{CDCl}_{3}\right)$ 和 ${ }^{13} \mathrm{C} \mathrm{NMR}\left(\mathrm{CDCl}_{3}\right)$ 数据见表 1.

化合物 2: 淡黄色油状, $[\alpha]_{\mathrm{D}}^{25}+46\left(c 0.11, \mathrm{CHCl}_{3}\right)$; HRESI-MS $m / z 493.3086[\mathrm{M}+\mathrm{Na}]^{+}$(calcd for $\mathrm{C}_{30} \mathrm{H}_{46} \mathrm{O}_{4}$ 470.3396). ${ }^{1} \mathrm{H} \mathrm{NMR}\left(\mathrm{CDCl}_{3}\right)$ 和 ${ }^{13} \mathrm{C} \mathrm{NMR}\left(\mathrm{CDCl}_{3}\right)$ 数据见 表 2.

\section{3 体外抗肿瘤活性测试}

肿瘤细胞购置于中山大学医学院, 按照文献[14], 采用 MTT 法测试甾醇对抗肿细胞的抑制率, 应用 SPSS 软件处理数据, 重复两次实验, 计算 $\mathrm{IC}_{50}$ 值.

\section{References}

[1] Si, M.; Zhan, X. T. Chin. J. Marin Drugs 2003, 6, 46 (in Chinese). (司枚, 展翔天, 中国海洋药物, 2003, 6, 46.)

[2] You, Y. C. J. Dalian Univ. 2001, 22, 29 (in Chinese). (由业诚, 大连大学学报, 2001, 22, 29.)

[3] Coll, J. C. Chem. Rev. 1992, 92, 613.

[4] El-Gamal, A. A. H.; Wang, S. K.; Dai, C. F.; Chen, I. G.; Duh, C. Y. J. Nat. Prod. 2005, 68, 74.
[5] Sarma, N. S.; Krishna, M. S.; Pasha, S. G. P.; Rao, T. S. Prakasa; Venkateswarlu, Y.; Parameswaran, P. S. Chem. Rev. 2009, 109, 2803.

[6] Wang, C. J.; Liao, X. J.; Xun, S. H. Nat. Prod. Res. Dev. 2010, 22 , 1 (in Chinese).

(王长军, 廖小建, 徐石海, 天然产物研究与开发, 2010, 22, 1.)

[7] Wang, C. J.; Liao, X. J.; Xun, S. H. Chem. Res. Appl. 2011, 23, 619 (in Chinese).

(王长军, 廖小建, 徐石海, 化学研究与应用, 2011, 23, 619.)

[8] Kelley, W. S. Bio/technology 1996, 14, 28.

[9] Holder, J. R.; Bauzo, R. M.; Xiang, Z.; Haskell-Luevano, C. J. Med. Chem. 2002, 45, 2801.

[10] Pramanik, D.; Majeti, B. K.; Mondal, G.; Karmali, P. P.; Sistla, R. J. Med. Chem. 2008, 51, 7298.

[11] Chi, B.; Park, S. J.; Park, M. H.; Lee, S. Y.; Jeong, B. Bioconjugate Chem. 2010, 21, 1473.

[12] Cheng, S. Y.; Wen, Z. H.; Wang, S. K.; Chiang, M. Y.; El-Gamal, A. A. H.; Dai, C. F.; Duh, C. Y. Chem. Biodivers 2009, 6, 86.

[13] Kingston, J. F.; Gregory, B.; Fallis, A. G. J. Chem. Soc., Perkin Trans. 1 1979, 2064.

[14] Diaz-Marrero, A. R.; Porras, G.; Aragon Z.; de la Rosa, J. M. J. Nat. Prod. 2011, 74, 292.

[15] Duh, C. Y.; El-Gamal, A. A. H.; Song, P. Y.; Wang, S. K.; Dai, C. F. J. Nat. Prod. 2004, 67, 1650.

[16] Alley, M. C.; Scudiero, D. A.; Monks, A. Cancer Res. 1988, 48, 589.

(Cheng, F.; Lu, Z.) 